\title{
Attractor reconstruction of an impact oscillator for parameter identification
}

\author{
Mukthar Sayah ${ }^{\mathrm{a}}$, Murilo Da Silva Baptista ${ }^{\mathrm{b}}$, James Ing $^{\mathrm{a}}$, Marian Wiercigroch ${ }^{\mathrm{a}, *}$ \\ ${ }^{a}$ Centre for Applied Dynamics Research, School of Engineering, Fraser Noble Building, King's College, \\ University of Aberdeen, Aberdeen, AB24 3UE, UK \\ ${ }^{b}$ University of Aberdeen, King's College, Aberdeen AB24 3FX, UK
}

\begin{abstract}
This paper presents a simple and novel approach, based on nonlinear time series analysis of an experimental system, to infer from subtle alteration of the system dynamics the changes caused in the system parameters. Using the acceleration time-series as a measurement of simulated and experimental impact oscillators (serving as a model for the drilling conditions with intermittent contact between the drill bit and the formation), the systems attractor is reconstructed and characterised. It is shown that the stiffness correlates with the topology of the reconstructed attractor. Non-impacting trajectories form an approximate plane within the three dimensional reconstructed phase-space, and contact with the constraint causes a systematic deviation from the linear subspace, the inclination of which, measured by the statistics of the tangent vector, can be used to infer the stiffness. This relationship between the topology of attractor and the stiffness was also verified experimentally. Based on the developed framework, it is now possible to classify the stiffness of the impacted material from a single variable in a simple way and in real time. Keywords: Impact oscillator, phase-space reconstruction, stiffness ratio, parameter identification, vibro-impact drilling.
\end{abstract}

\footnotetext{
${ }^{*}$ Corresponding author
}

Email addresses: m.sayah@abdn.ac.uk (Mukthar Sayah), murilo.baptista@abdn.ac.uk (Murilo Da Silva Baptista), j.ing@abdn.ac.uk (James Ing), m.wiercigroch@abdn.ac.uk (Marian Wiercigroch) 


\section{Introduction}

Systems with impacts have received a great deal of attention both in the study of fundamental non-smooth dynamics and in engineering applications. Periodic impacts of colliding bodies in mechanical systems such as gears can be highly detrimental to their life span, and can generate vibration and noise. In contrast, some other mechanical systems rely on impact motion to perform their intended function. An example of such systems is percussive drilling, where higher impact performance is required to increase the penetration rate through hard rock formations. Hence, advanced understanding of impacting systems should help in prolonging their usable life span by controlling vibration and noise levels and/or to improve their performance and functionality.

Impacting systems have been studied extensively in the literature mainly assuming a harmonically forced oscillator having rigidly or flexibly constrained vibration amplitude. In both cases, the systems exhibit periodic and complex behaviour including chaos. Peterka and Vacik [1] studied numerically impact system behaviour. Time-series recordings, phase-space trajectories, and Poincaré maps were used to determine parameters that led to stable periodic motion and also to explained the bifurcations that led to chaotic behaviour for different impact conditions. In [2] Peterka studied non-smooth characteristics arising from mechanical system with impacts. Several types of non-smooth interactions were considered during the soft impact. Nonlinear analysis techniques were used to study how parameters affect the system behaviour and the modifications suffered in the dynamics of the system under the influence of changing impact stiffness. The results showed that the smoothness of the trajectories in phase space is lost in a boundary surface representing the moment of the impact, when both the vector field and the Jacobian of the system become discontinuous. Pi̊st and Peterka [3] analysed the dynamical properties of a single degree-of-freedom mechanical system with a soft restraint using Hertz contact theory. They emphasized that the applied model based on the Hertz contact law could describe the real behaviour of impact systems better than other models, because it respects the nonlinearity of the restoring force between the impacted bodies. System parameters were studied and the phase-space portraits showed an enlargement of the trajectories as impact stiffness was increased and the energy lost during the impacts is increased with 
increase of the impact velocity. In Di Bernardo et al. [4] a non-smooth dynamical systems theory was developed based on approaches from bifurcation theory to classify the complex behaviour of impacting dynamic systems. A coherent framework for studying dynamic behaviour of piecewise-smooth and hybrid systems was presented and discussed with numerous examples of relevant technical developments and application from numerical and experimental results.

Thompson and Ghaffari [5] studied an impact oscillator rebounding elastically whenever the relative displacement drops to zero. Using numerical methods they showed cascades of period doubling bifurcations leading to chaotic regimes. Shaw and Holmes [6] studied a model of a periodically forced oscillator with a flexible constraint and analysed its corresponding bifurcations. A mapping was constructed from the model and used to investigate the dynamic impact forces for a nonlinear oscillator with a single discontinuity. Later, Shaw $[7,8]$ studied the harmonic and sub-harmonic double impact motions for a system having two sided amplitude constraints. Bifurcation theory was employed to study the impacting responses and their stability including unstable symmetric orbits with two impacts. Whiston [9] studied the steady state responses of a harmonically excited piecewise linear oscillator using a dynamical systems approach. They constructed from numerical simulations Poincaré maps to follow the evolution of the impact with time. Nordmark [10] took the analysis of impact system further and used it to study the response of a single degree-of-freedom oscillator with a hard impact. The results have been derived from a system with instantaneous impact. The author has utilised the phase-space geometrical representation of the system to study different impact conditions. Analytical methods were developed to construct one-dimensional map between the different phase-space sections to study the singularities and stability of the orbits caused by different impact conditions. A square root singularity in the Jacobian matrix was found to appear exactly at the grazing impact conditions.

Extensive studies have been carried-out at the Centre for Applied Dynamic Research (CADR), the University of Aberdeen, on the dynamical behaviour of the impact systems. Different sizes experimental rigs were used to study the behaviour of drill-string where the rotation and axial excitation were combined to drill vertical and horizontal holes on 
real rock formations. Comprehensive studies had also been carried out by the CADR on a piecewise linear oscillator system impacting against elastic beam. The impact oscillator experimental rig was originally developed by Wiercigroch and Sin $[11,12]$ to study bifurcations of a system with a two sided restraint. Later, Ing et al. [13] modified it by removing one constraint to examine experimentally the bifurcation scenarios of near grazing conditions. Their study was supported by a robust mathematical model developed for the same setup. Later, the same authors [14] conducted experimental and semi-analytical studies on the behaviour of the impact oscillator under different exciting parameters. Smooth and non-smooth bifurcations were observed experimentally and explained using mapping solutions. Pavlovskaia et al. [15] studied the behaviour of a linear oscillator constrained by an elastic beam. Several bifurcations for near grazing conditions were observed experimentally. The results showed that the attractor evolution is influenced by an interplay between smooth and non-smooth behaviour.

Even though there is an extensive literature on nonlinear analysis to characterise the behaviour of nonsmooth dynamical systems, there is a need for experimental methods to help determining the moment, the conditions, and the parameters of the impact and of the impacted material, topics that constitute the main purpose of this work. Nonlinear time series analysis will be applied in here to investigate the underlying properties of this impacting mechanical systems.

There are several examples in the literature explaining the application of nonlinear time series analysis to study the behaviour of engineering systems. Craig et al.[16] applied phase-space reconstructing techniques and measured the fractal dimension to monitor a dynamical system with bearing clearance. Their system was representing an elastically supported rotor excited by imbalance and restricted to two dimensional movements. The study was conducted on three different magnitudes of clearance using two different shaft speeds. Initially, conventional embedding delay method was used to reconstruct the phase-space for time series recorded in one direction but, it resulted in bad quality and unacceptable reconstructions leading to substandard computation of correlation dimension. This was reasoned to be a consequence of the weakening of the coupling between the two motions in the two dimensions. Also, increasing the magnitude of the clearance 
caused more information lost about each direction. A modified method to reconstruct the phase-spaces was then used by considering values from other observation recorded from the system into the reconstructing vector. The study showed that the computed correlation dimension decreases as the magnitude of clearance is increased. This was observed in each case of the shaft speed. The authors emphasised the importance of using correlation dimension techniques on monitoring events related to the mechanical system.

Da Silva et al. [17] discussed the application of the nonlinear time series analysis to study experimental data when drilling in a semi-insulating Gallium arsenide, GaAs sample. They used the method of false nearest neighbours to estimate the embedding dimension of the attractor. The correlation dimension and maximum Lyapunov exponent were computed for the reconstructed phase-space to obtain more understanding of the dynamics of the experimental system in order to develop models for numerical simulations. A noteworthy feature of their article is that all of the computations were performed using a special-purpose time series analysis software called TISEAN [18]. Reiss and Sandler [19] also applied nonlinear time series analysis to electronic signals generated by a synthesizer to observe harmonic structures over long time spans. The study concluded that the nonlinear time series analysis techniques have a tendency to identify predominantly short term dynamics. Piiroinen et al. [20] studied a single degree-of-freedom system representing horizontally excited pendulum restricted by a rigid stopper. The time series showed a sudden change to chaotic and period-adding cascade behaviours as a parameter is altered, which is the expected dynamics that could be predicted from a grazing bifurcation normal form. These results were also achieved from the numerical simulation of the system, where discontinuity map was applied to derive the coefficient of the square-root normal-form map. Numerical continuation method was also applied to found the grazing periodic orbit and the linearization of the system along the orbit. Then the system parameters were computed, which allow changing in the system behaviour to chaos or period-adding cascade. The study also highlighted the important role of the damping coefficient in the observed dynamics, and the importance of using correct amplitude in order to get close quantitative agreement between the numerical and experimental results. This implies that the amplitude might require an adjustment to overcome the effects of 
the parameters that might not be considered in the model. Kodba et al. [21] have studied a signal of a RLC-diode circuit driven by an alternating voltage source of variable amplitude. Using systematically nonlinear time series analyzes the author observed that as the strength of the voltage source is increased, the system reaches a chaotic state through a period doubling route. Perc [22] also applied the same techniques mentioned in the previous papers to study the dynamic of electrocardiogram and human gait systems [23]. The study emphasised that the time series recoded for a human gait possesses deterministic chaotic nature which was consistent with other related studies.

Detecting an impact between the mass and the restraint, and its stiffness from experimental data is not an easy task especially if the impact is near grazing and also if the noise level was high. The experimental setup may require making use of a more sophisticated sensing system, thus more complicated and sensitive devices would be needed. Ing [25] and Ing et al. [13] investigated the determination of the times of the impact between mass and restraint, where a threshold in the mass acceleration was applied to distinguish between the impact data. The authors have also used the velocity-acceleration and the displacement-acceleration phase planes to identify the impact size and magnitude instead of using the standard displacement-velocity phase portraits.

This article is intended to study the behaviour of an impacting system using nonlinear time series technique to estimate the mechanical parameters, in particular the stiffness of the impacted restraint (and consequently its mechanical properties) based on the geometric representation of the time (the reconstructed phase-space). In Section 2, the experimental setup and sensing devices are briefly described. Section 3 presents the data handling process and standard nonlinear time series analysis and the phase-space reconstruction process. Section 4 goes on to explain several approaches that were developed to estimate the stiffness of the impact from acceleration time histories.

\section{Experimental setup}

The experimental rig used in this article to generate the time histories is the same rig as detailed in [24]. It is a piecewise linear impact oscillator system with constrained amplitude in the form of an elastic beam. In previous studies the focus was on determining 


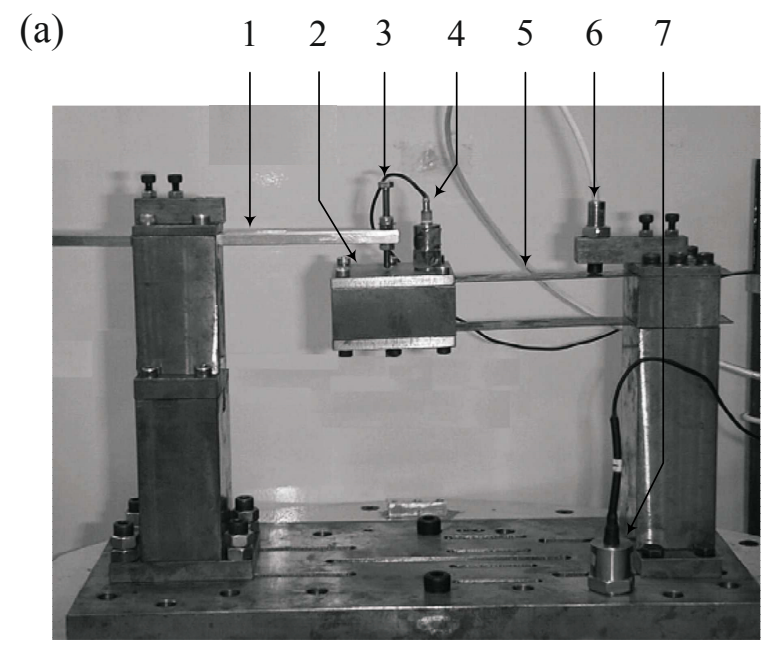

(b)

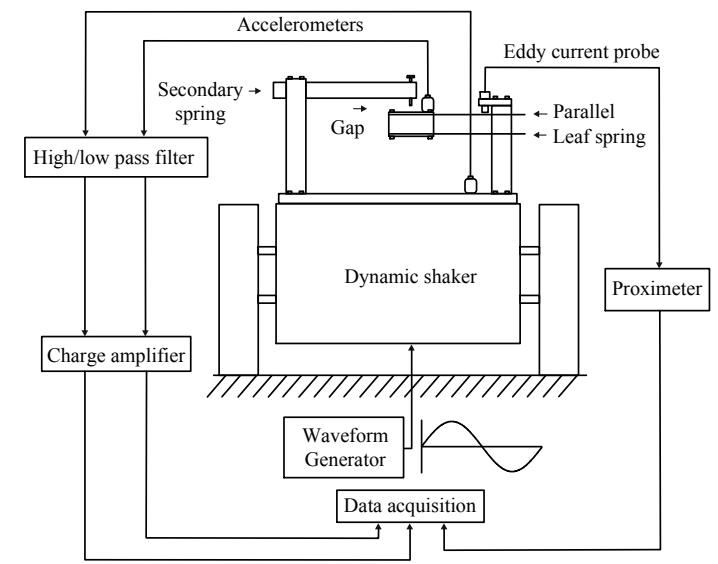

Figure 1: (a) Photograph of the experimental setup: 1-elastic beam; 2-moving mass; 3pin; 4-mass accelerometer; 5-leaf springs; 6-eddy current probe; 7-base accelerometer,(b) Schematic of the experimental setup including the measurement system, Adopted from $[13]$.

the bifurcation structure of near grazing trajectories as a function of excitation frequency and forcing amplitude. The intention of the current study will be on developing a framework to determine the material effective stiffness from reconstructed phase-space of single output time histories. As shown in Fig. 1(a) the setup consists of two parallel columns made from steel with different heights mounted on a steel plate mounted at the top of electro-dynamic shaker. A steel block mass is attached to two parallel leaf springs attached to the short column. The leaf springs here provide primary stiffness for the system which determined by the springs length. The other column is used to support an elastic beam and bolt, which is separated from the top side of the mass by a pre-defined gap $(g)$. This beam provides the secondary stiffness in the system. It can also be controlled by the length of the beam.

An eddy current displacement transducer is attached to the top of the short column pointing towards the leaf springs to measure the relative displacement of the moving mass. It is denoted as $x_{m}$ and the signal is passed to the computer through a proxymeter and interface panel. There are also two accelerometers; one is mounted on the top of the moving mass and another one is mounted on the base plate. They are denoted as $\ddot{x}_{m}$ and 
$\ddot{x}_{b}$, for the moving mass and the body, respectively. Both accelerometers were connected to high/low pass filters and charge amplifiers, and then through the interface panel as shown in Fig. 1(b) to the computer. The signals were recorded in real time via Labview data acquisition system.

A controlled sinusoidal signal was used to drive the system. The contact between the pin and the mass occurs when the relative displacement of the moving mass is equal to zero. The tests were carried out by varying the excitation parameters and monitoring the system responses until even a subtle change was observed. Then the responses were recorded for different values of the forcing amplitude and exciting frequency for situations in which the attractors remain topologically similar [25]. The time histories were then smoothed using Savitzky-Golay algorithm by fitting a second order polynomial curve to preserve the sharpness and height of peaks of the signal in time domain, whilst minimising the effect of noise as much as possible.

\section{Direct method of identifying impacts stiffness}

As was mentioned previously, the trajectory in the phase-space of the non-smooth system crosses the boundaries of adjacent regions to form an additional plane. The size and inclination of the trajectory in the additional plane is influenced by the impact stiffness. Constructing acceleration-displacement phase portraits for different time histories generated by the same parameters but with different stiffness ratios showed that the in-

clination and the size of the trajectory in the additional plane increases as the impact stiffness increased. Fig. 2(a) is an example of this relationship.

However, in the presence of parameter noise, especially in the gap, this relationship became less clear. Additionally, in real drilling applications only a single measurement might be possible. The obvious solution is to reconstruct the phase-space from just one variable using the embedding theorem. Fig. 2(b) shows the projection of $x_{t}-x_{t+2 \tau}$ planes from the reconstructed phase-spaces for the same time histories. As can be noted, the planes inclination-stiffness relationship is still preserved in the reconstructed phase-space.

The advantage of using reconstructed phase-space over the one formed from the original variables is that the analysis is done in a single variable with no need to rely on the 
(a)

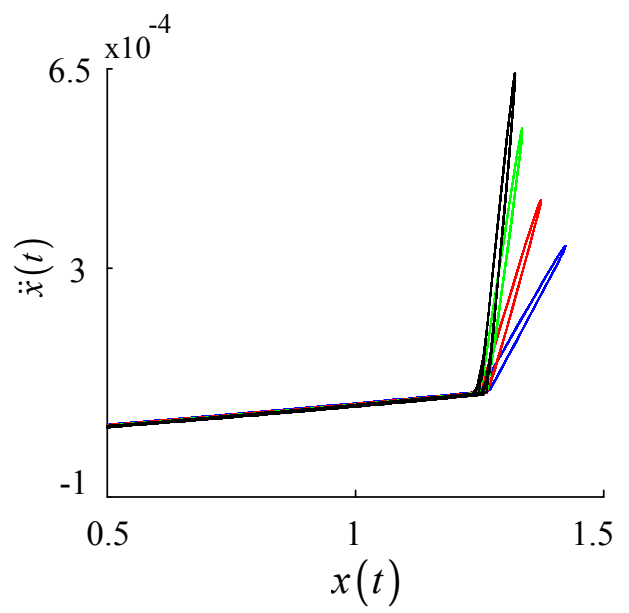

(b)

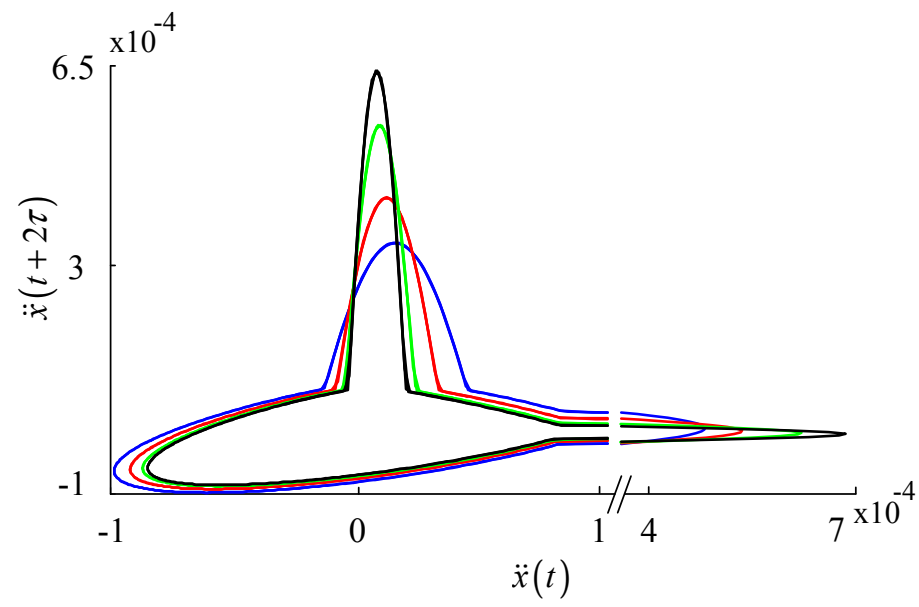

Figure 2: (a) Acceleration- displacement phase portrait for theoretical time histories with stiffness ratio of 15 (blue); 29 (red); 60 (green); and 90 (black), (b) $x_{t}-x_{t+2 \tau}$ planes of reconstructed phase-space for the same time histories.

accuracy, synchronicity, and other features of all the other measurements. Also, in the original phase-space some properties could be hidden by the effect of the parameters noise. Several time histories have been analysed in this paper including time history recorded for no impact response, near grazing impact and larger impact. This study has considered the acceleration time histories of mass because of the ability show sudden changes on the contact force. Samples of recorded mass acceleration response for both impact and no impact response are shown in Fig. 3(a, b).

Since, the analysed time histories in this paper are generated from a well-known system, there is no need to apply any techniques to classify the impact or non-impact data. However, in real applications not all the system parameters are known. Hence, there is a need for a technique to distinguish between these two types of data. To detect the impact time histories Ing et al. [13] used a threshold index in the mass acceleration time history plots. Additionally, the velocity-acceleration and displacement-acceleration phase portrait were used, an example of which is shown in Fig. 3(b) and 2(a). A simpler way to detect the impact is to compute the second derivative for the mass acceleration time histories. Fig. 3(c) shows an example of the numerically computed derivative of the mass acceleration presented in Fig. 3(b). As it can be seen the sharp impact spikes presented 
(a)

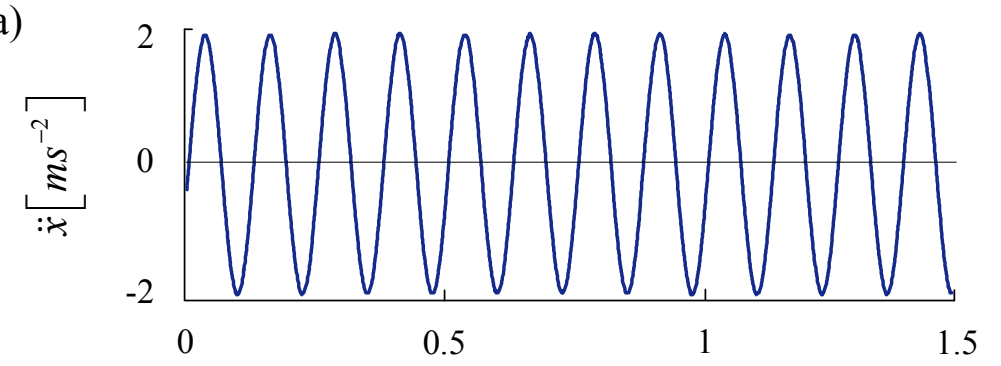

(b)
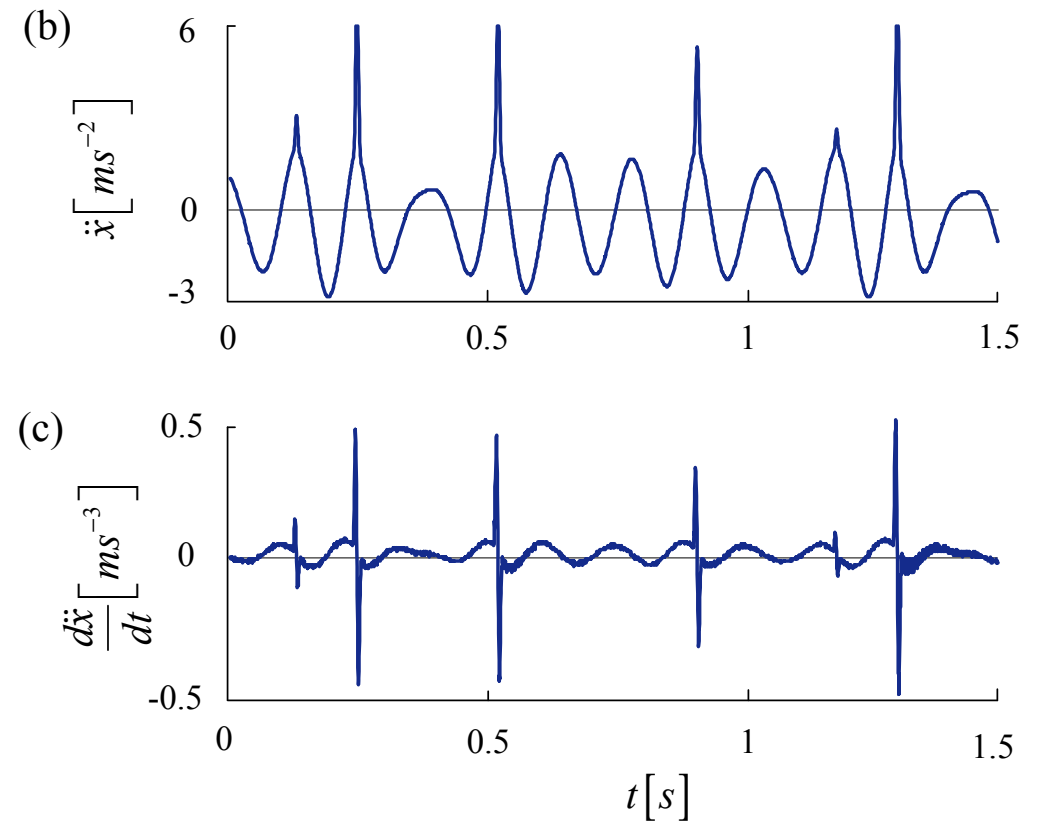

Figure 3: Samples of the recorded mass acceleration time histories for (a) Data with no impact, (b) data with impact, (c) Calculated derivative of the mass acceleration time history shown in (b).

on the acceleration time history are now well pronounced and easy discernable from the other data. Even the near grazing and insignificant spikes can be well observed from the derivative. Another technique to detect the impact times is to compute and plot the root mean square of the acceleration time history. This is done by calculating the mean of all data points and then subtracting it from each point separately and computing its square product. This technique separates all the impact points from the non-impact points in the time series. 


\section{Time series analysis}

In experimental studies the governing equations of motion for the system are usually unknown and all that is available from the system is a set of observations in form of time histories. There is no information about the underlying system dynamics and its complexity or sensitivity to the initial conditions. One way for getting better understanding of the system dynamics and their properties is to apply nonlinear time series analysis, where the analysis is done with just a single or a set of time histories.

The process of applying the nonlinear analysis is usually commenced by reconstructing the phase-space from one or a set of time history to visualise the system dynamics and unfold its attractor, for which then the stationarity and determinism requirements of the underlying system are verified. Finally, some nonlinear measures such as the Lyapunov exponents and correlation dimensions are computed for the characterisation the attractor.

\subsection{Phase-space reconstruction}

Phase-space reconstruction is a geometrical representation of the trajectories described by the time histories with similar topology to the phase-space constructed from the original variables. The reconstruction is performed from a single data set because the variables in a deterministic system are related to each other [26]. Takens in [27] proved that the reconstructed attractor has similar topological and dynamical properties to the original attractor. This theorem is known as Taken theorem of phase-space reconstruction and it states that for a compact manifold $M$ with dimension $m$, its generic property of $\Phi_{\varphi, v}: M \rightarrow$ $\mathbb{R}^{2 m+1}$ can be defined as:

$$
\Phi_{\varphi, y}(x)=\left(y(x), y(\varphi(x)), \ldots \ldots . y\left(\varphi^{2 m}(x)\right)\right)^{T},
$$

where: $v$ are smoothed vector field, $x(t)$ corresponds to the values of measurements taken from the dynamical system at the state $t \in M$, and $\varphi$ is the flow of the vector field.

In the phase-space reconstruction two important parameters have to be considered, which are embedding delay $(\tau)$ and the embedding dimension $(m)$. A proper embedding delay can be estimated by using the mutual information method or auto-correlation function, and a proper embedding dimension can be estimated from the false nearest neighbour method [18]. 


\subsubsection{Embedding delay}

Two criteria should be fulfilled to obtain a proper embedding delay, where the first is that, the delay $\tau$ has to be large enough to enable obtaining relevant and significantly different information from variable $x$ at time $t+\tau$ and variable $x$ at time $t$. The second criterion is that $\tau$ should not be too large so that the system does not lose the memory of its initial state and the new variable becomes random with respect to the first variable. The idea here is to create a set of variables that are neither highly decorrelated nor highly correlated to each other. A proper embedding delay can be estimated by determining the time for which auto-correlation function decays firstly by half. It can also be estimated by computing the first decay of the auto-mutual information [28]. The absolute value of the difference between the maximum and minimum points in the time series is first computed and then partitioned into equal intervals. According to [28] the mutual information, $I$ is then calculated as follows:

$$
I(S, Q)=\int P_{s q}(s, q) \log \left[P_{s q}(s, q) / P_{s}(s) P_{q}(q)\right] d s d q,
$$

where: $S$ and $Q$ are variables, $P_{s}$ and $P_{q}$ are the probabilities of finding the variable $S$ and $Q$ in the $s-t h$ and $q-t h$ bin, and $P_{s q}$ is the joint probability distribution function of $X$ and $Q$. In our case, the variable $\mathrm{S}$ represents the variable $x(t)$ and the variable $\mathrm{Q}$ the time-delay variable $x(t-\tau)$.

Mutual information takes into account the nonlinear correlations of the data. Hence, it is usually used for chaotic data more than the auto-correlation algorithms, which is usually considered as good rule of thumb for time histories that are nearly regular or not chaotic.

\subsubsection{Embedding dimension}

A proper embedding dimension can be estimated using the false nearest neighbour method. The technique assumes that the attractor for a deterministic system can be folded and unfolded smoothly with no sudden irregularities in its structure. This implies that any two close points in the reconstructed embedding space should remain sufficiently close in any forward time iteration. Thus, the distance between points $p(i)$ and $p(t)$ which 
are a small amount, $\epsilon$, apart in the reconstructed attractor should remain close under a sufficiently short forward iteration and the distance should not grow further than the threshold $R_{t r} \epsilon$. Hence, any point that is near the point $t$ and does not fulfil this criterion will be considered as a false nearest neighbour to the point $t$. The fraction of points having a false nearest neighbour has to be minimized by selecting a sufficiently large $m$. Trajectory crossings that create false neighbours induced by the choice of a low value of $m$ result in the loss of information about all other variables that influence the measured variable [29]. In this article, Coa's algorithm [30] has been used to determine whether a point $y_{n(i, d)}(m)$ is a false neighbour of $y_{i}(m)$. It will be a false neighbours if $a(i, m)$ is larger than the threshold.

Let us compute the false nearest point $a(i, m)$, which is expressed as following:

$$
a(i, m)=\frac{\left\|y_{i}(m+1)-y_{n(i, d)}(m+1)\right\|}{\left\|y_{i}(m)-y_{n(i, d)}(m)\right\|},
$$

where: $\|$.$\| is Euclidian distance between the points of the index i, y_{i}(m+1)$ is the reconstructed vector of embedding dimension $m+1$, the point $y_{n(i, d)}(m)$ is nearest neighbour to the point $y_{i}(m)$ in the dimension $m$ of the phase-space.

Fig. 4 shows the process of phase-space reconstruction for three different time histories, starting from selection of the time histories, estimating the embedding delay and embedding dimension, and finally, the reconstructed phase-space. The time histories presented in the figure are the acceleration response for three different cases of impact conditions namely, no-impact, near grazing impact, and larger impact. The data was generated for the system parameters of excitation frequency $=7.9 \mathrm{~Hz}$, forcing amplitude $=0.25 \mathrm{~mm}$, natural frequency $=9.38 \mathrm{~Hz}$, Damping coefficient $=1.3 \mathrm{kgs}^{-1}$.

In the first case there was no contact between the restraint and mass, hence the system is linear. In the embedding parameters determination, the first minimum mutual information was found around $60 \tau$ and the saturation of percentage of false nearest neighbour corresponded to an embedding dimension of 3 . In the reconstructed phasespace the attractor exhibits periodic behaviour and forms a circular orbit within one plane. In the other two cases there was contact between the secondary stiffness and moving mass, near grazing impact in the second case and larger impact in the third 
(a)

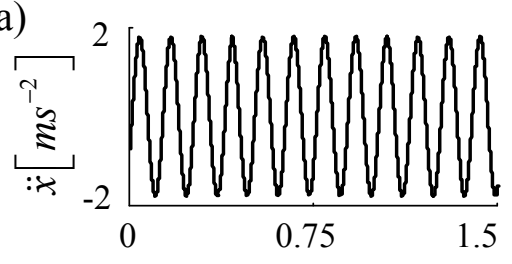

(b)

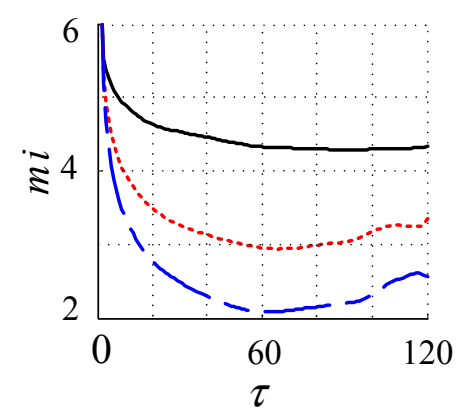

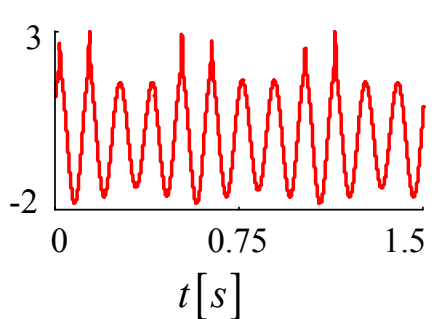

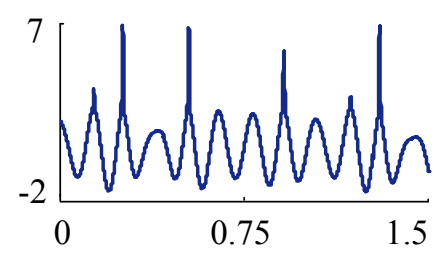

(c)

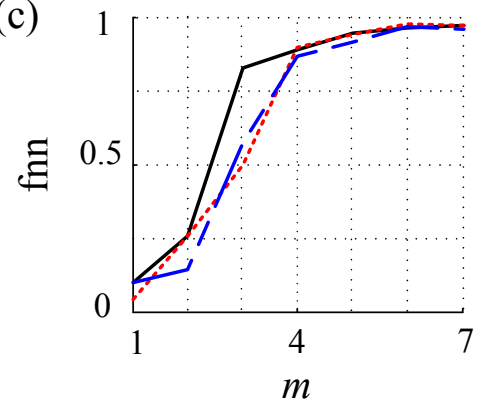

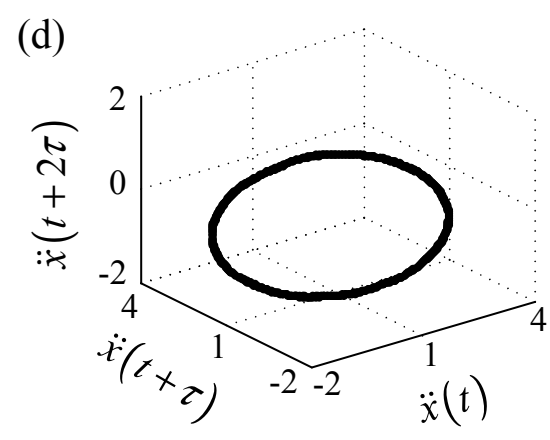
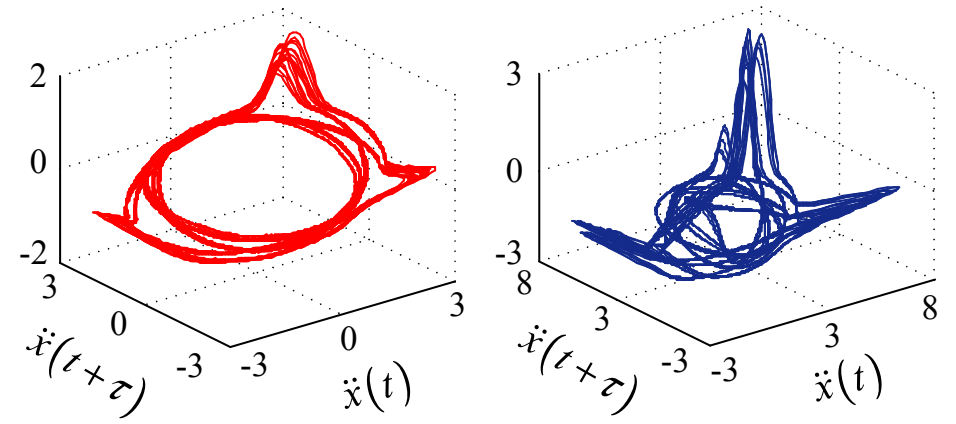

Figure 4: (a) Acceleration time history for non-impact case (solid black); near grazing impact case (dotted red); and larger impact case (dashed blue), (b) Determination of embedding delay by mutual information technique. The first minimum mutual information is found around $60 \tau$, (c) Determination of embedding dimension by false nearest neighbour. The percentage of the false nearest neighbour are more saturated with $80 \%$ corresponding to $3 \mathrm{~m}$ for non-impact case and $4 \mathrm{~m}$ for the other two cases,(d) Reconstructed the phase-space for the three cases using embedding theorem. 
case. In determining the embedding parameters, the first minimum mutual information for both were found also around $60 \tau$ and the saturation of percentage of false nearest neighbour corresponded to $4 \mathrm{~m}$ implying that the system becomes more complicated as compared with the first case and it will require at least 4 dimensions to be described. In the reconstructed phase-space, the attractors also exhibited periodic behaviour and formed a circular orbit in one plane with three groups of spikes attached to the circular orbit, but they cross the boundaries of the linear plane to another plane.

\subsection{Stationarity and determinism}

Reconstructing a proper phase-space is not only necessary to perform further nonlinear time series analysis, but it is also important to verify that time series comes from a system that is stationary and deterministic. Even though the studied system might not be fully deterministic, determinism is a major component as will be demonstrated in the following sections. Identifying its deterministic dynamics allows to identify some change in the system being observed, a change that points to how the environment of the drilling is changing.

\subsubsection{Stationarity test}

The stationarity of the data is confirmed by computing the cross-prediction error. This technique checks for predictability of the data with itself by using the data segments to predict the value of another forward segment [31]. The time history is first partitioned into non-overlapping segments $j$ with a sufficient equal length. Then each segment of data is used to make prediction for another one. For every point $p(t)$ in the first segment, the closest neighbour $p(i)$ from the next segment has to be found and the average value

neighbourhood are then used to predict the value of $x_{i+\delta t}$, which is the future values of $x_{i}$. The average prediction error is then used to evaluate the accuracy of the obtained predictions.

Finally, the whole procedure is repeated for all combinations of $j$ and $i$, and then a plot of those combinations is performed. If the obtained $S_{j i}$ was significantly larger than the average this means that the stationarity requirements for the examined data are not fulfilled. Fig. 5 shows an example of cross-prediction error plotted for data with large 


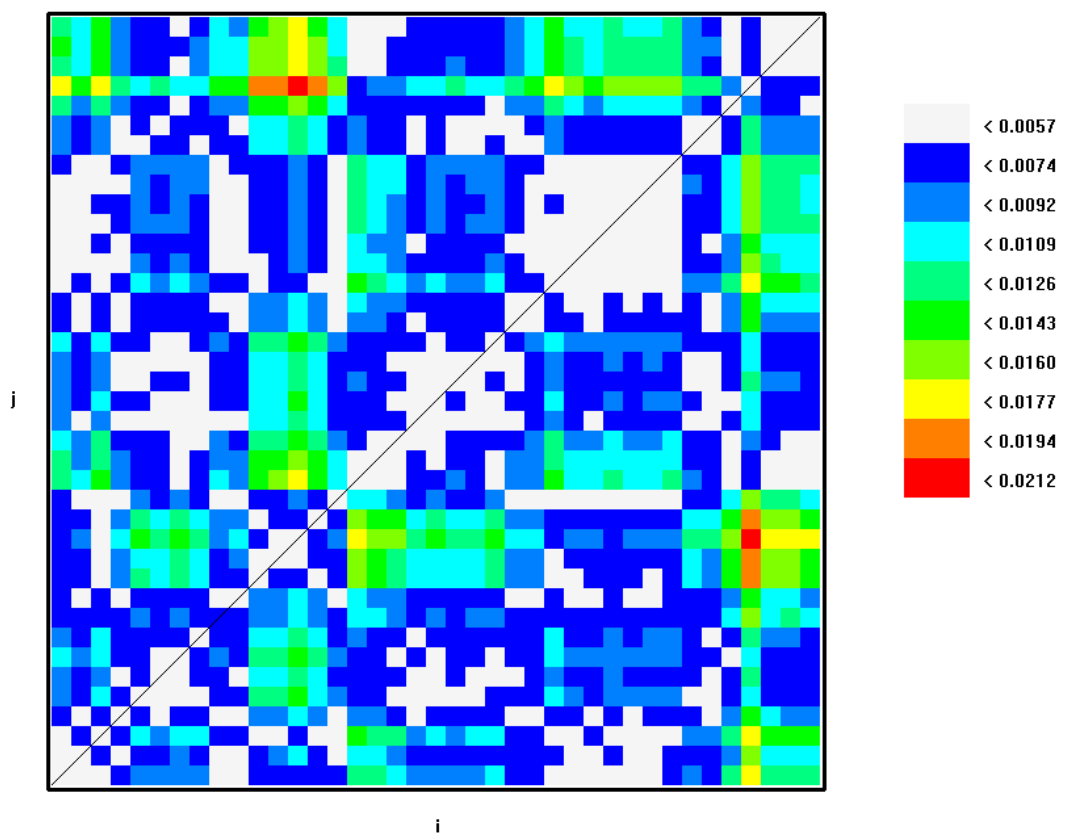

Figure 5: Confirming the stationarity of the data using cross-prediction error. $\mathrm{N}=100000$, neighbour distance 0.1 and factor of increasing the neighbour distance 1.4142. Minimum and maximum errors are 0.1058 and 0.1872 .

impact case. The maximum and minimum cross-prediction errors are 0.1058 and 0.1872 , respectively. Since the different between them is not even one time larger and all the errors differ maximally by a factor less than 0.05 , it is clear this data originates from a stationary process.

\subsubsection{Determinism test}

This test enables confirmation of the presence of deterministic chaos in the examined data and distinguishes it from irregular random behaviour. The determinism test used also to produce true vector filed for the analysed data. The Kaplan and Glass method [32] is used here to assess the determinism of the embedded data. The method requires construction of an approximate vector field of the reconstructed phase-space, partitioned into coarse grained boxes with equal dimension as the embedding space. A vector is then assigned to each box occupied by the trajectory where every time $i$ the trajectory passes through the box $k$, will generates unit vector $e_{i}$ and its direction is determined by direction of the phase-space points where the trajectory enters and leaves the box. 
(c)

(a)

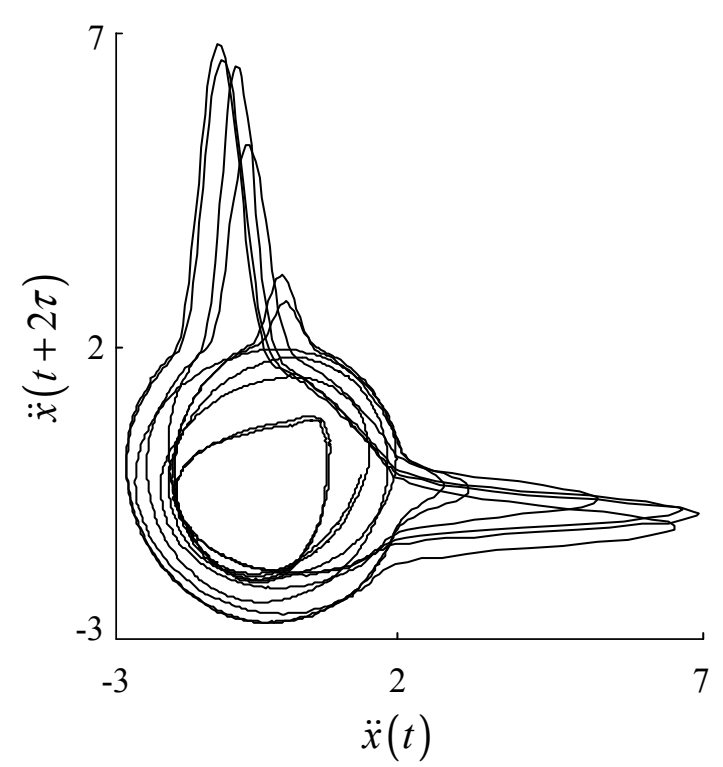

(b)

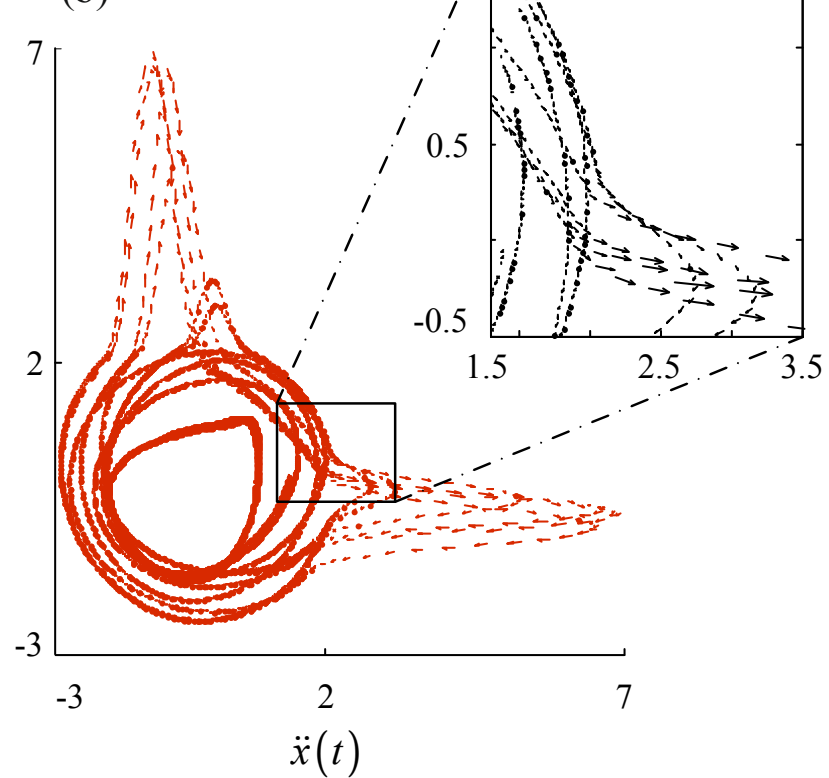

Figure 6: Determinism test; (a) the reconstructed phase-space for $c 8$ data set, (b) the approximated factor field. $N=100000, \tau=59$, and $m=4$. The determinism factor is 0.91, (c) zoomed area from the approximated factor field.

Approximation of the vector field $V_{k}$ for each box is computed as the average vector of trajectories passing through the box. Performing this task with all the boxes occupied by the trajectory provides the directional approximation of the system vector field and its uniqueness depends on the direction of all the vectors in each box. The length of vector field increases if all vectors inside the boxes are pointing into the same direction. This implies a unique vector field, hence, a deterministic system. In contrast, presence of trajectories crossing inside the boxes will decrease the length of $V_{k}$, indicating a non-uniqueness in the vector field. Since $V_{k}$ is normalised, the determinism factor for a completely deterministic system is 1 , whilst for a random walk it is 0 . Fig. 6 shows the reconstructed phase-space and its approximated vector field for data with larger impact case. The pertaining determinism factor is 0.91 , which is close to the uniqueness of the vector field 1. According to Kaplan and Glass [], the analysed data is generated for deterministic system. 
By comparing the three reconstructed phase-spaces, it can be easily observed that the attractor of the non-impact time history lies within the same plane. Whilst, in the other two reconstructed phase-space the attractor lives in same plane as far as there is no impacts and then escapes to another plane after impacts occurs. Fig. 7 shows a comparison between the three reconstructed phase-spaces. As indicated by the dimension estimation, the non-impacting trajectory lies in an $m-1$ subspace of the reconstructed phase-space. Impacts perturb the system into full $m$ dimensional space. However, even in the full nonlinear system, the non-impacting part of the response remains largely in the subspace of the linear system. This fact will be used to help the impact detection.

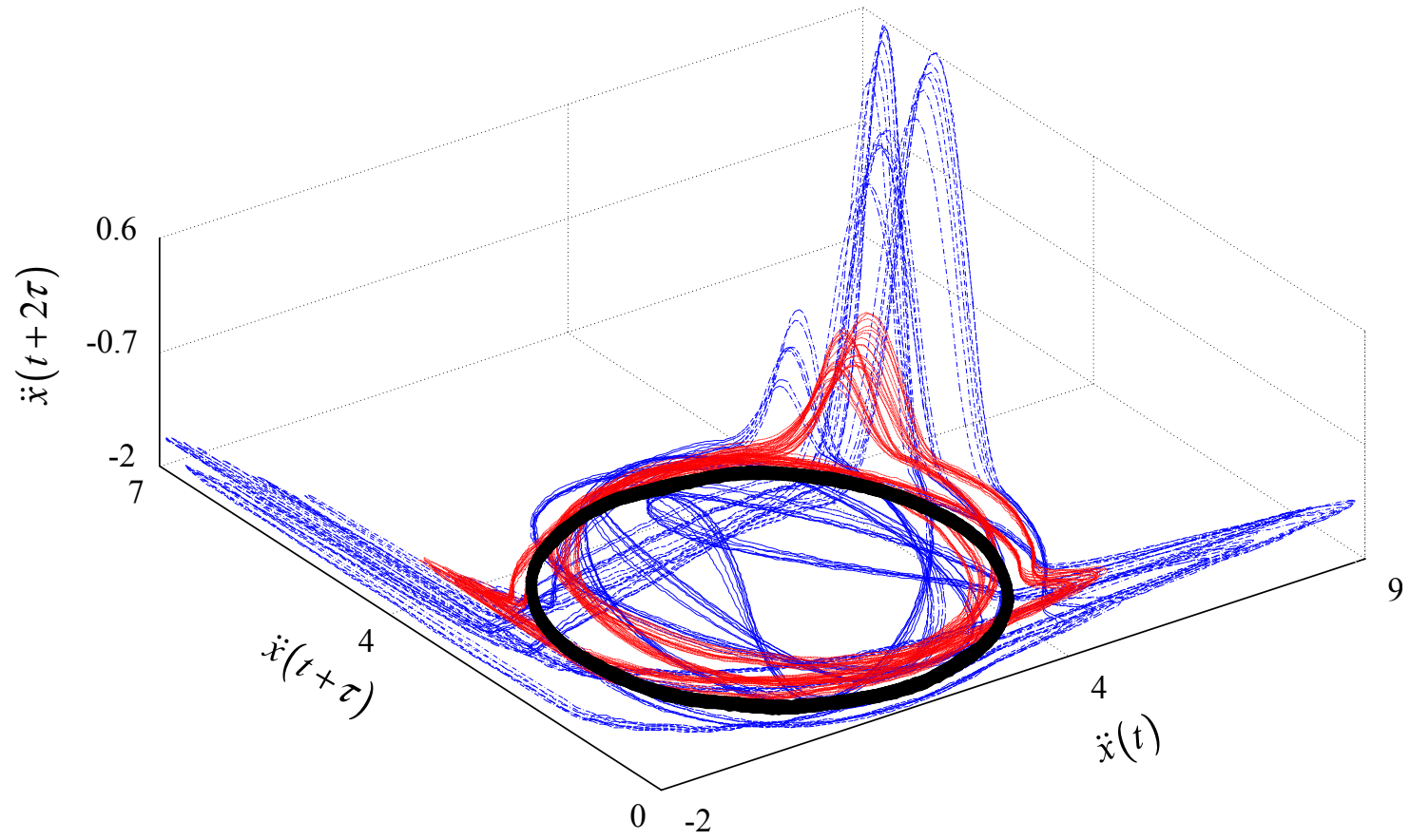

Figure 7: Comparison between three reconstructed phase-spaces for non impact (solid black), near grazing impact (solid red), and larger impact (dashed blue).

This relationship becomes clearer if the reconstructed phase-space is projected into $x-z$ plane. An understanding of this relation could provide a key concept of identifying the stiffness of the impact from just studying the dynamical behaviour of the system. 


\section{Impact parameter identification}

The application of nonlinear time series analysis is extensively used to study dynamical systems in many branches of science and understanding their underlying dynamical behaviour. Yet, this is the first attempt to use such analysis to study impact system parameters, specifically related to down-hole drilling. This article is intended to propose a framework based on applying nonlinear time series method to determine mechanical properties related to the rock formation while being drilled. The main concept behind the proposed analysis is to use the variation of trajectory inclination from the plane of the linear system to the other plane, caused by impact. The effect of changing stiffness on the orientation of the plane will be investigated. For this purpose the inclination of the secondary plane in the reconstructed phase-space is computed. This approach has utilised common angle estimation techniques, namely, the gradient and least square approach.

\subsection{The new concept of stiffness identification}

As mentioned previously, the attractor is restricted to a subspace of the phase-space during no contact mode, then once the contact occurs the attractor escapes from the "linear plane" to a nonlinear surface within the phase-space. This escape is found to be a result of the secondary stiffness term in the equation of motion, which explains the presence of another plane in reconstructed phase-space. The attractor remains in the linear plane while there is no contact. However, as soon as there is contact the trajectory moves out into the "nonlinear plane" before it moves back again to the linear one at the end of contact. Also, it was mentioned previously that the inclination of the second plane depends upon stiffness magnitude. As the stiffness increases, the inclination of the second plane increases and the angle of the plane becomes sharper compared with lower stiffness. Thus studying the relationship between plane inclination and impact stiffness could reveal more information about the impact stiffness and also develop an index for the stiffness.

The process of computing the inclination of the plane is undertaken by applying a second order polynomial filter (Savitzky-Golay) to scale the data and minimise the noise effect as much as possible. The purpose of smoothing the time histories at this stage 
is because it is easier to fit a filter in a one dimensional data than in a vector. Also, it will preserve the shape and magnitude of impact spikes and original coordinates of tangent vectors. The standard phase-space reconstructing process is then performed for the filtered time history after estimating embedding parameters as was described earlier. Also the stationarity and determinism requirement have to be fulfilled as described earlier.

In order to estimate the inclination of the plane from embedded data, the following procedure is used. The tangent vectors $\vec{V}_{t}$ are first computed for each variable from the embedded data by calculating the difference between the coordinates for a given distance, which should be sufficiently large and will depend on the sampling rate and the magnitude of the generated vector. The tangent vector is given by $\vec{V}_{t}=\left(V_{x}^{t}, V_{y}^{t}, V_{z}^{t}\right)$, where

$$
V_{x}^{t}(N-n)=x(N)-x(N-n)
$$

and

$$
V_{y}^{t}(N-n)=y(N)-y(N-n)
$$

and

$$
V_{z}^{t}(N-n)=z(N)-z(N-n)
$$

where: $N$ is element of the time history and $n$ is determined by the sampling rate, and the amount of averaging required.

There is an increase of the moving mass acceleration after the impact compared with its original acceleration before the impact. Accordingly, values of the tangent vector products of the reconstructed phase-space will be different before and after the impact. Tangent vectors after the impact will have a greater magnitude than tangent vectors before the impact. This fact is exploited to separate the contact and non-contact planes. A histogram is used to assess the distribution of resultant tangent vectors. Based on the difference of the moving mass acceleration, there will be two classes of distributions. Higher distribution with lower tangent vector magnitudes presents the non-contact plane, while a lower distribution, but with higher tangent vector magnitudes presents the contact plane. Based on this definition a cutoff magnitude can be determined visually from the histogram in the point where the two distributions overlap. Fig. ?? shows the process of separating planes using tangent vector magnitude. 
(a)

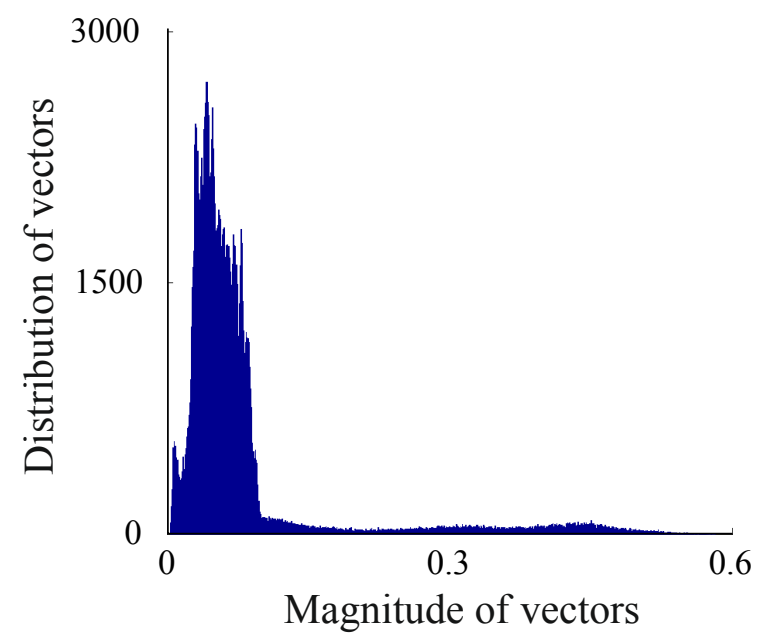

(b)

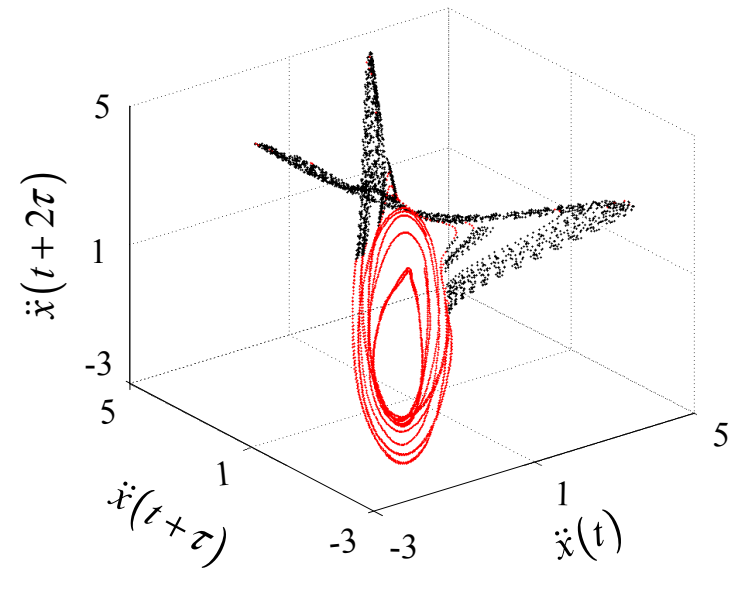

Figure 8: Separating contact from non-contact data; (a) histogram of the tangent vectors magnitude, (b) embedded data after separating non-contact (dot red) from contact data (dot black).

The vector products are then projected into $x-z$ plane and the inclination is computed as the inverse of gradient of this plane by considering $x$ and $z$ coordinates from the embedded data for each plane. Averaging of the angles in each orbit before and after the impact is then performed and used to create a coloured map for the angles by plotting angles before the impact against angles after it for all the time histories. The inclinations of the planes are computed by the gradient approach. Assuming that $V_{l}$ and $V_{n}$ are tangent vectors in linear and nonlinear planes, respectively. Such that,

$$
\vec{V}_{l}=\overrightarrow{a_{1} i}+\overrightarrow{b_{1} j}+\overrightarrow{c_{1} k}
$$

and

$$
\vec{V}_{n}=\overrightarrow{a_{2}} i+\overrightarrow{b_{2} j}-\overrightarrow{c_{2} k}
$$

The two vectors are then projected into the $x-z$ plane, and inclination of the contact $\left(\theta_{l}\right)$ and non-contact $\left(\theta_{n}\right)$ planes can now be computed as

$$
\theta_{l}=\tan ^{-1}\left(\overrightarrow{c_{1} k} / \overrightarrow{a_{1} i}\right)
$$

and

$$
\theta_{n}=\tan ^{-1}\left(\overrightarrow{c_{2} k} / \overrightarrow{a_{2} i}\right)
$$


The other approach to compute the inclination of the nonlinear plane is to calculate it directly as coefficients of a polynomial fitted curve. The embedded data within the impact region are first defined using the tangent vectors magnitude distribution as discussed in the previous approach. Then a polynomial regression approach is applied to fitted two curves through the embedded data before and after the impact region and the gradients of these curves are direct measures of the planes inclination.

\subsection{Applying stiffness identification method}

The previous section discussed the framework of stiffness identification from the reconstructed attractor of the impact oscillator. An example was given to demonstrate the adopted methodology and explain how the computation was done. The adopted framework was then tested on numerical time histories and then on numerical time histories with added noise to demonstrate its effectiveness in noisy data. Finally, it is applied to experimental time histories. This section presents the data processing and manipulating and discusses some results obtained from applying the adopted framework into several time histories.

\subsubsection{Applying stiffness identification method to numerical data}

The time histories analysed in this section are all obtained from the impact oscillator setup, which is described in [14]. For the simplicity of the analysis, that data is required to be obtained from the same system parameters, apart from varying the stiffness ratios. Hence, only one parameter is varying each time. Therefore, co dimension one bifurcations are obtained each time, such as period doubling. This was confirmed via comparison of the phase portraits and construction of bifurcation diagrams. According to Ing et al. [14] The mathematical model of the impact oscillator is expressed as following:

$$
\begin{gathered}
x^{\prime}=v, \\
v^{\prime}=a \omega^{2} \sin (\omega t)-2 \xi v-x-\beta(x-e) H(x-e),
\end{gathered}
$$

where: $x$ is the displacement, $v$ is the velocity, $t$ is the time, $\beta$ is the stiffness ratio, $e$ is the gap, $a$ is the forcing amplitude, $\xi$ is the damping ratio, and $\mathrm{H}$ is the Heaviside step function. 

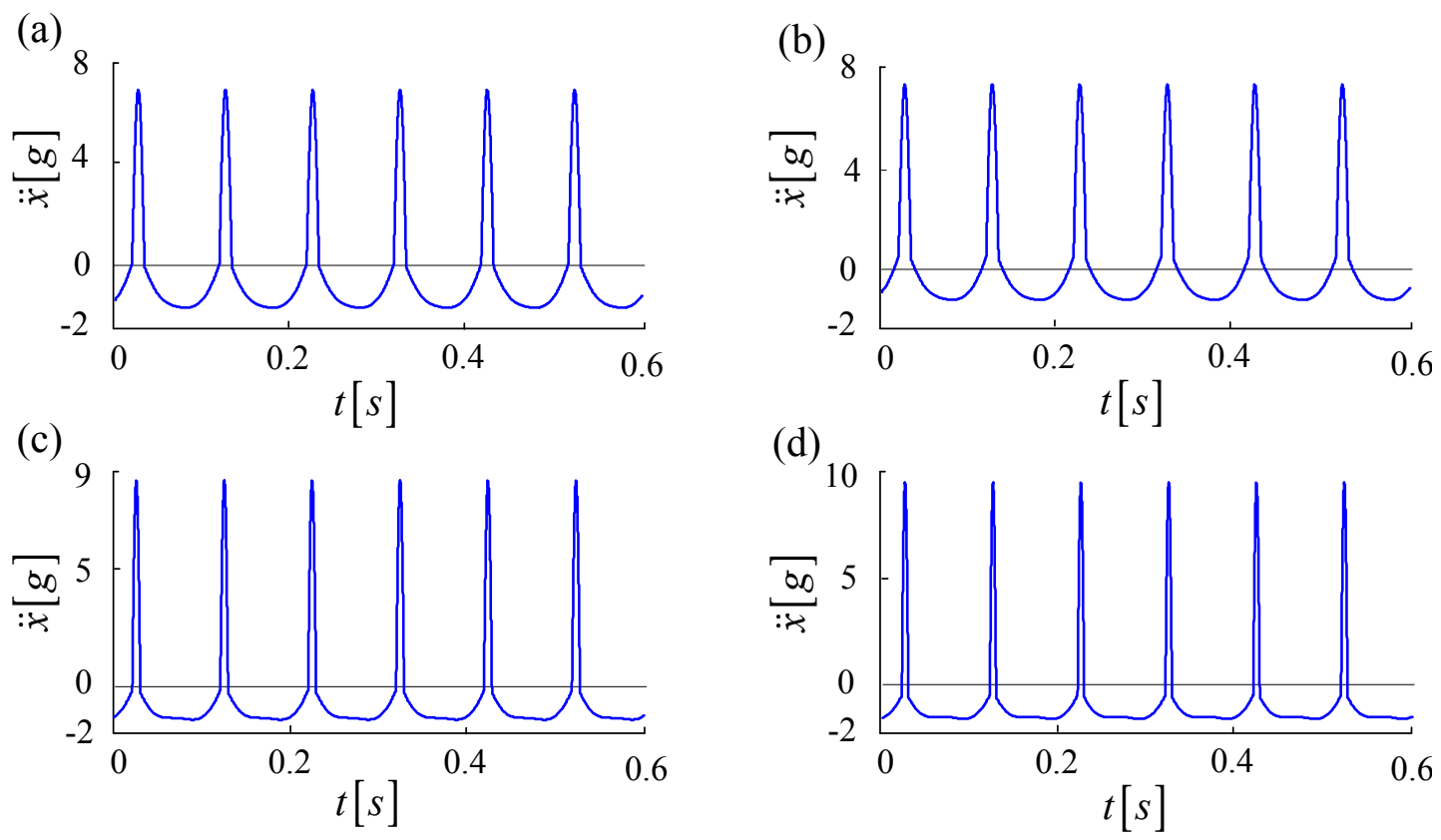

Figure 9: Samples of the numerical acceleration time histories for stiffness ratios; (a) $\beta=12$, (b) $\beta=26$, (c) $\beta=31$, (d) $\beta=47$.

The above differential equation was solved numerically in Matlab using the "ODE45" solver. Time histories were generated and phase portraits were then constructed to assess the system behaviour. Bifurcation diagram was also constructed to study the effect of changing stiffness ratio on the system behaviour. The parameters of the mathematical model were chosen as follows: $a=1.9, \omega=1.05, f_{n}=9.48 \mathrm{~Hz}, \xi=0.02$, and $e=2.8$. Seventeen stiffness ratios were considered, 5, 10, 12, 15, 20, 26, 31, 35, 40, 47, 50, 55, 60, 80, 90, and 100. Fig 9 shows four examples of the time histories obtained from the simulation for stiffness ratios $\beta=12,26,31$, and 48 . As it can be observed, the magnitude of the acceleration spikes is increasing and the duration of the impact is decreasing as the stiffness ratios are increased.

The phase-space reconstruction process was performed as discussed earlier to determine the embedding parameters. Minimum mutual information and false nearest neighbour techniques were used to estimate the embedding delay and embedding dimension for each time history. The first minimum mutual information was found around $17 \tau$ and the false nearest neighbour percentage was saturated around $80 \%$ which corresponded to $4 \mathrm{~m}$. Following the estimation of the embedding parameters, the attractor reconstruction 


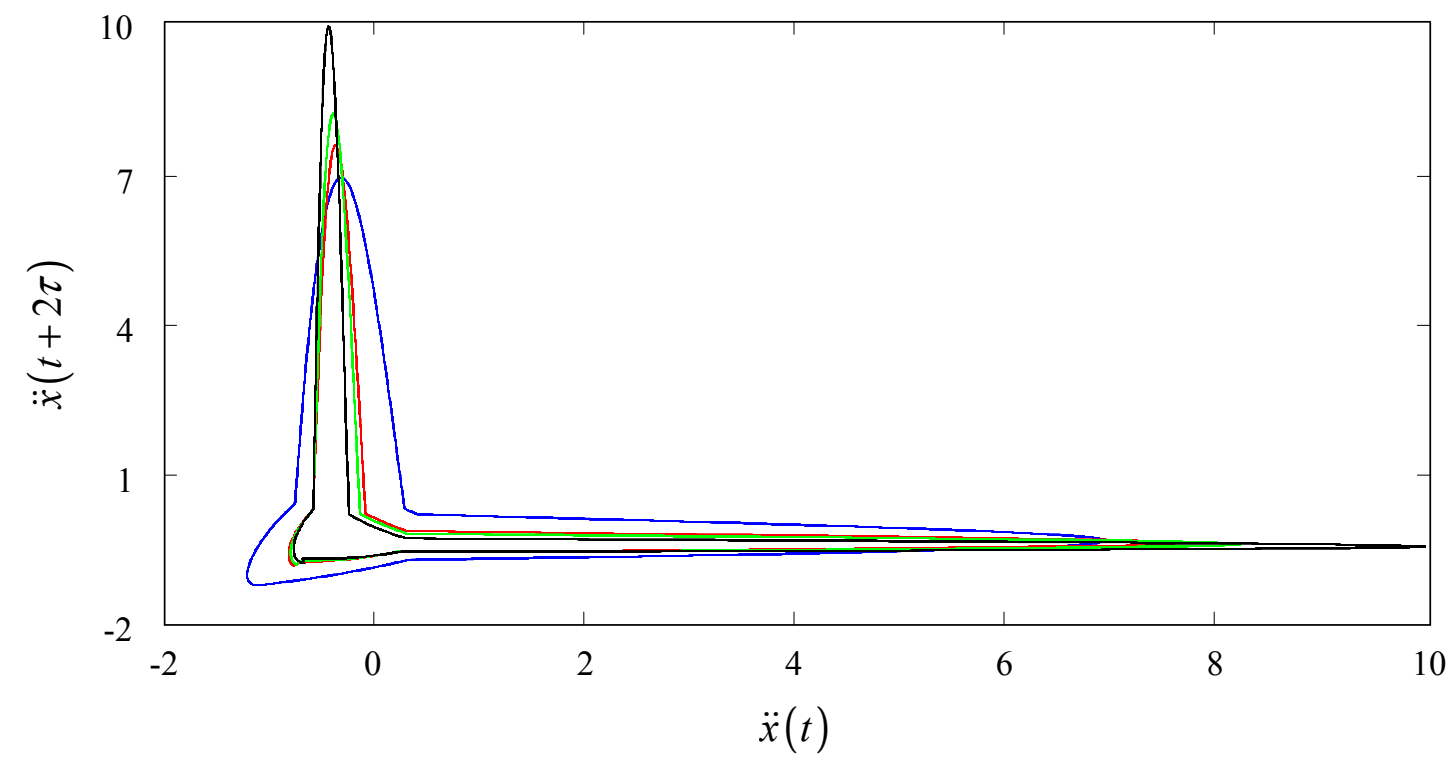

Figure 10: Reconstructed phase-space for the numerical time histories from for stiffness ratios; (a) $\beta=12$ (solid blue), (b) $\beta=26$ (solid red), (c) $\beta=31$ (solid green), (d) $\beta=47$ (solid black).

was performed for all time histories. Fig 10 shows the reconstructed phase-space for the four numerical time histories cases projected in $x-z$ plane. As can be observed from the figure, the attractor forms a circular orbit in one plane and then when contact occurs the attractor escapes to another plane. The size of the attractor in the $z$ plane increases and its duration decreases as the stiffness ratios increases. The inclination of the contact plane increases as stiffness ratio increases.

The inclination of the contact and non-contact planes were also computed as discussed earlier. The results of inclination estimation using the gradient approach are presented in the coloured map on Fig 11. It is obvious from the map that the inclination-stiffness relationship forms a clear trend in which the inclinations of planes before and after impact are increasing as the stiffness ratio increases. Separation between the inclinations is not the same for all cases. The separation decreases with increase of the stiffness ratios until it reaches stage where different between the inclinations became very minor and hard to distinguish. This can be noted clearly from the different between stiffness ratio cases 60 , 80, 90, and 100, where increment of the inclination before and after the impact became very close. 
The results of applying a polynomial fit to the four stiffness ratios presented in Fig 9 are illustrated in table 1 . The table confirms that higher stiffness ratios produce higher inclination before and after the impact, which confirms the results of inclination-stiffness trend obtained from the gradient approach.

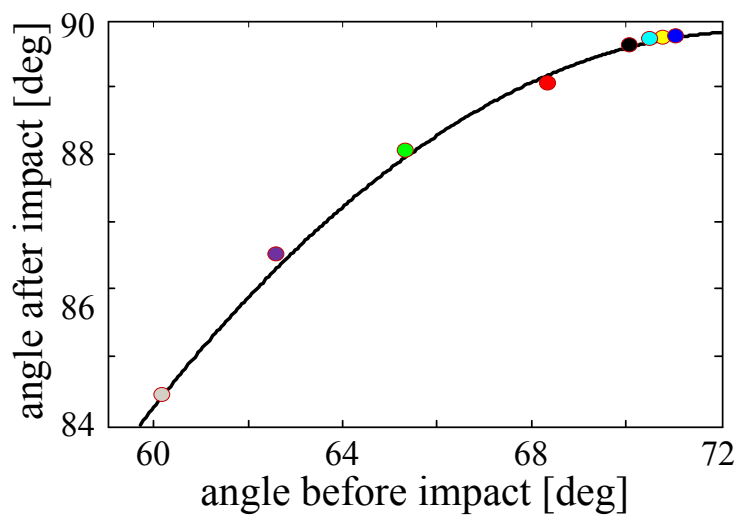

Figure 11: Plot showing the planes inclination before and after the impact calculated for all the theoretical time histories with stiffness ratios of 5 (gray), 20 (sea-green), 35 (green), 50 (red), 60 (black), 80 (cyan), 90 (yellow), 100 (blue), and the black line denotes theoretical angles trend.

Table 1: Results of applying polynomial fit to the reconstructed phase-space for four numerical acceleration time histories.

\begin{tabular}{ccc}
\hline Stiffness ratio & $\begin{array}{c}\text { Inclination before impact } \\
\text { (degree) }\end{array}$ & $\begin{array}{c}\text { Inclination after impact } \\
\text { (degree) }\end{array}$ \\
\hline 12 & 61.3 & 85.2 \\
26 & 62.9 & 86.8 \\
31 & 63.2 & 87.3 \\
47 & 67.4 & 88.4 \\
\hline
\end{tabular}

\subsubsection{Noise effect on the stiffness-inclination relationship}

As mentioned earlier, noise could hide some properties related to the system deterministic behaviour and affect the performance of the proposed framework and its accuracy 
and ability to distinguish between different stiffness ratios. For this purpose, two different noise scales cases were investigated by adding a Gaussian noise to four numerical time histories with different stiffness ratios.

Following the process of reconstructing the phase-space, the inclination of contact and non-contact planes was computed for four time histories with stiffness ratios $=12,26$, 31, and 47 and noise less than $5 \%$ of the forcing amplitude. For the other cases the noise is $9 \%$ of the forcing amplitude. The results of applying the proposed framework in two both cases are presented on the coloured maps in Fig 12(a, b). From the two maps, it is clear that the stiffness-inclination relationship is still preserved. A higher stiffness ratio cases produce higher planes inclinations. For results shown in Fig 12(a), where a lower noise is applied, the inclination of the planes are still clustered in groups and the stiffness-inclination trend is still well pronounced with a clear separations between each stiffness. In Fig 12(b) where higher noise is applied, the inclinations of the planes became more scattered especially before the impact. This is due to the fact that the noise has greater effect non-contact data where the distance between data points are more closed due to the lower acceleration of the moving mass. Although, the planes inclinations of the contact data are a bit scattered, there is still a clear separation if higher difference between stiffness ratios is considered. This can be observed from the stiffness ratio cases 26 and 31.

\subsubsection{Applying stiffness identification method to experimental data}

After confirming the ability of the proposed framework to distinguish between different stiffness ratios for the numerical data, it is vital at this stage to check its ability in the experimental data generated from the impact system. For this purpose four acceleration time histories with a period doubling have been generated for stiffness ratios $\beta=12,26$, 31 , and 47.

The stiffness ratios were computed from the primary and secondary stiffness of the experimental rig. Secondary stiffness was computed from the natural frequency of the system, whilst the primary one was estimated from static test using Instron machine. Fig 13 shows samples of the experimental acceleration time histories. As it can be observed from the figure again that the magnitude of the acceleration spikes is increasing and the 
(a)

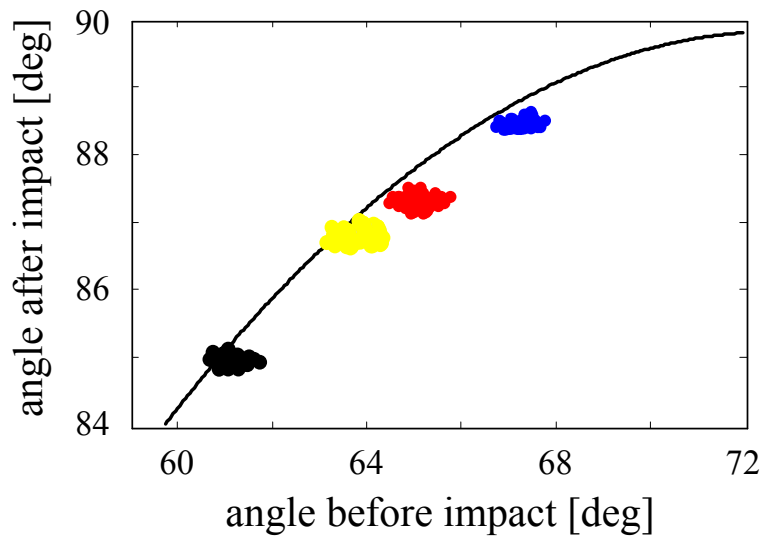

(b)

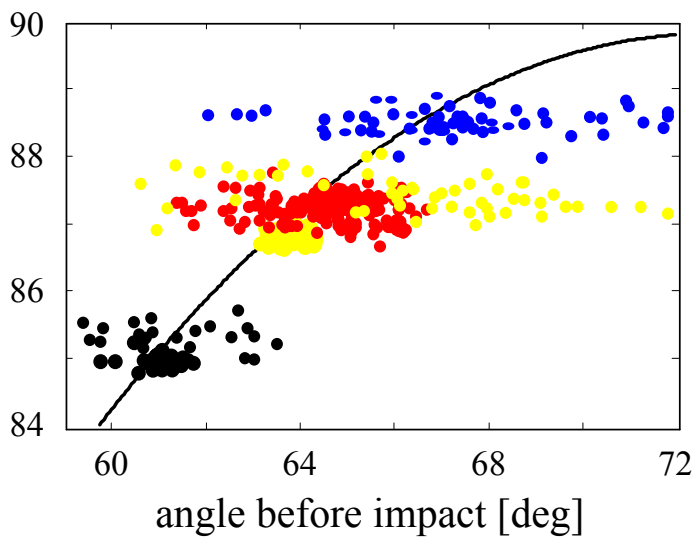

Figure 12: Plot of plane inclinations calculated for two noise scale cases for stiffness ratios of 12 (dot black), 26 (dot yellow), 31 (dot red), 47 (dot blue), and the black line denotes theoretical angles trend; (a) noise smaller than 5\% of the forcing amplitude, (b) noise larger than $9 \%$ of the forcing amplitude.

duration of the impact is decreasing as the stiffness ratios increases, which is similar to the numerical data presented earlier.

After smoothing the data by applying the second order Savitzky-Golay filter to all time histories, the process of estimating the embedding parameters and reconstructing the attractors repeated again for each case. Fig 14 shows the reconstructed phase-space for the four cases projected in $x-z$ plane. Again, the size of the attractors in the $z$ plane and their inclination depend on the stiffness ratio. A notch in impact region is noted in the attractors of higher stiffness cases, which is due to smoothing process. To overcome this effect a larger contact region should be considered when computing the inclinations.

The process of computing planes inclinations is performed again to estimate inclinations of contact and non-contact planes for all cases. The results are illustrated in the plot in Fig 15 where the inclinations estimated for experimental time histories are presented with stiffness-inclination trend obtained previously from the numerical data. As can be noted, the inclinations computed from the experimental data are clustered in groups widely spread and looks more separated from each other. Although, the experimental data was associated with noise, the inclination-stiffness trend are still preserved and well pronounced. Higher stiffness ratio case from experimental data produces also 

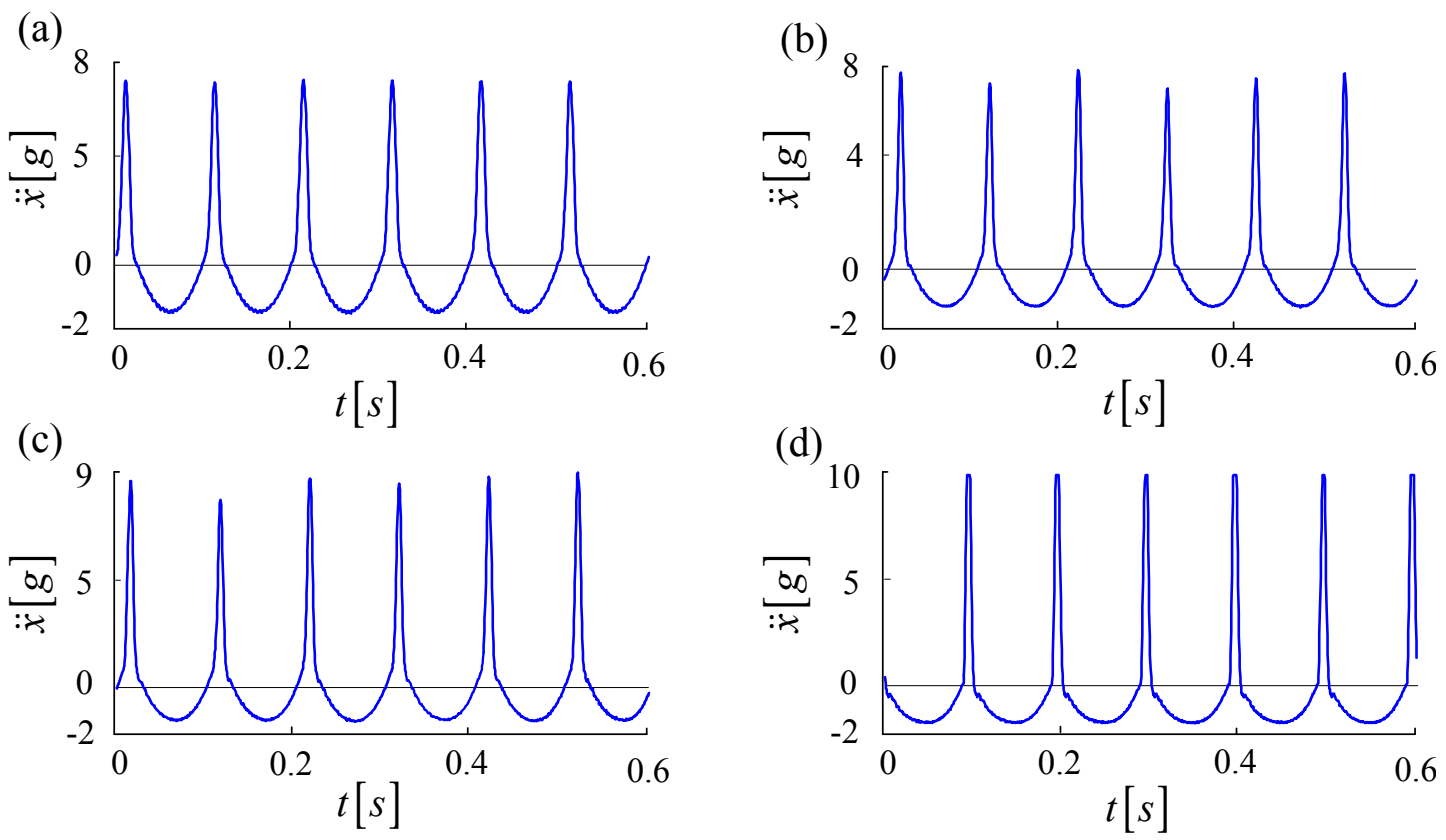

Figure 13: Samples from experimental acceleration time histories for stiffness ratio of;(a) $\beta=12$, (b) $\beta=23$, (c) $\beta=31$, (d) $\beta=48$

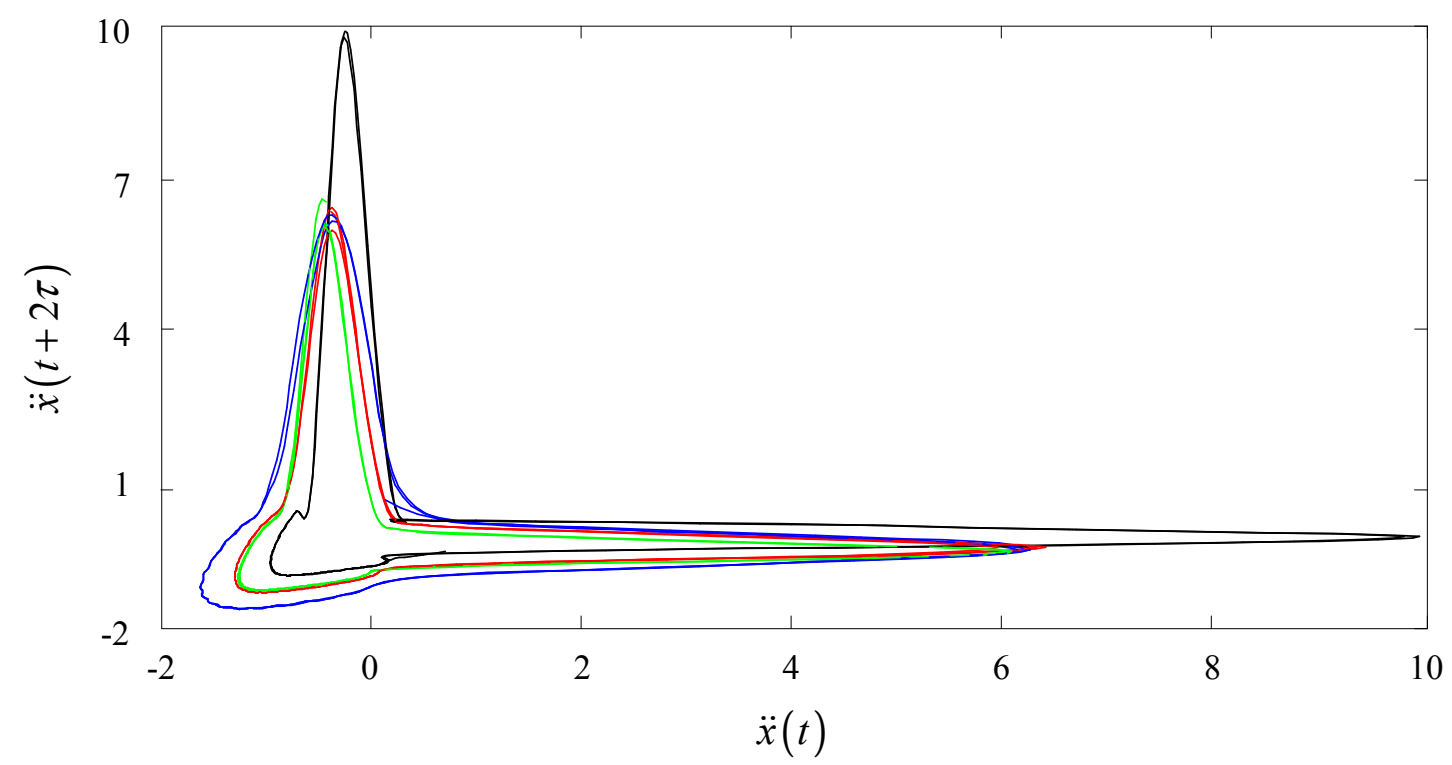

Figure 14: Reconstructed phase-space for experimental time histories for stiffness ratios; (a) $\beta=12$ (solid blue), (b) $\beta=26$ (solid red), (c) $\beta=31$ (solid green), (d) $\beta=47$ (solid black). 
higher inclinations similar to the numerical with clear separation between each inclination.

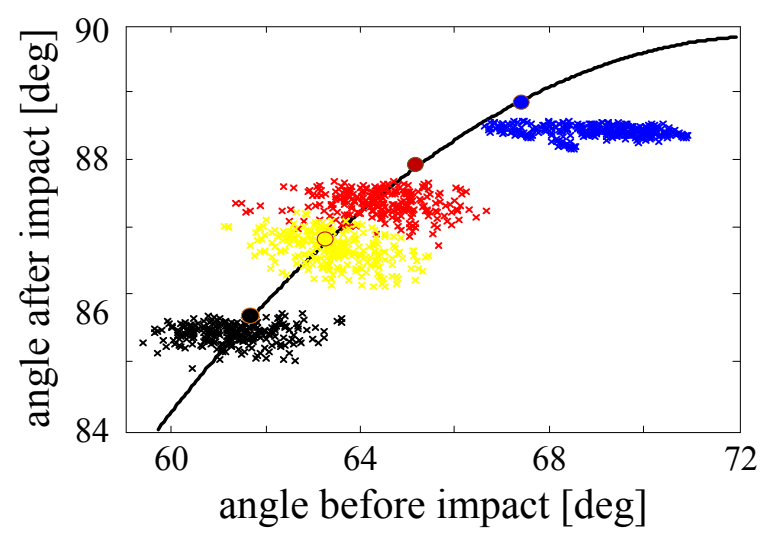

Figure 15: Comparison between the planes inclination before and after the impact calculated for experimental time histories for stiffness ratios of 12 (cross black), 26 (cross yellow), 31 (cross red), 47 (cross blue), and numerical time histories for stiffness ratios of 12 (dot black), 20 (dot yellow), 35 (dot red), and 50 (dot blue).

\section{Conclusions}

This work offers a simple and novel time-series analysis approach to characterise the reconstructed attractor of simulated and experimental impact oscillators, models for certain drilling conditions. To ascertain that the topology of the systems being studied are examples of dynamical systems, stationarity and determinism tests were computed. This is most relevant to the determination of the dynamical and topological properties of the reconstructed attractor, since the time-delay embedding approach used to reconstruct the attractor is well defined for deterministic and stationary systems.

The reconstructed phase-space for no-impact time histories showed that the attractor lives within a linear plane, whilst for the impact time histories the attractor escapes from the linear plane after the impact and then returns back. The deviation of the trajectories of the impact oscillator from the plane is larger, the larger the effective stiffness. Experimentally, the effective stiffness parameter can be controlled by adjusting the length of the elastic beam, and it represents the strength of the impacted material. Numerically, stiffness is a control parameter that can be changed by varying the parameter $\beta$. Therefore, 
the effective stiffness and the stiffness of the numerical model studied correlates with the angular deviation.

The reconstructed tangent vectors from both numerical and simulated time-series are then employed to measure this deviation. Firstly, their magnitude is used to identify the moment of impact. Larger (smaller) magnitudes correspond to data after (before) impact. Secondly, the angle between trajectory points after and before the impact is estimated by two methods. In the first method, this angle is estimated as an average of the inclination of embedded points before and after impact set with respect to the plane. In the second method, the angle is estimated from the angular separation of two fitted polynomials to the trajectory points in a $2 \mathrm{D}$ projection, each polynomial fitting data from either before or after the impact. Using the numerical model, it is shown that the relationship between stiffness and the topology of the attractor is noise invariant when the noise level is less than $5 \%$ of the amplitude value. However, at higher levels of noise, there is a slight uncertainty introduced into the stiffness estimation.

\section{References}

[1] F. Peterka, J. Vacik, Transition to chaotic motion in mechanical systems with impacts, J Sound Vib, 1992, 154(1):95-115.

[2] F. Peterka, Behaviour of impact oscillator with soft and preloaded stop, Chaos Soliton Fract, 2003, 18(1):79-88.

[3] L. Pi̊st, F. Peterka, Impact oscillator with Hertz's model of contact, Meccanica 2003, 38(1):99-116.

[4] M. Bernardo, C. Budd, AR. Champneys, P. Kowalczyk, Piecewise-smooth dynamical systems: theory and applications, Springer, London, 2007.

[5] JMT. Thompson, R. Ghaffari, Chaos after period-doubling bifurcations in the resonance of an impact oscillator, Phys Lett A, 1982, 91(1):5-8. 
[6] SW. Shaw, P. Holmes, A periodically forced piecewise linear oscillator, J Sound Vib, 1983, 90(1):129-155.

[7] SW. Shaw, Dynamics of a harmonically excited system having rigid amplitude constraints, PART 1: Subharmonic motions and local bifurcations, J Appl Mech-T ASME, 1985, 52(2):453-458.

[8] SW. Shaw, The dynamics of a harmonically excited system having rigid amplitude constraints, II: Chaotic motions and global bifurcations, J Appl Mech-T ASME, 1985, $52(2): 459-464$.

[9] G. Whiston, Global dynamics of a vibro-impacting linear oscillator, J Sound Vib, 1987, 118(3):395-424.

[10] AB. Nordmark, Non-periodic motion caused by grazing incidence in an impact oscillator, J Sound Vib, 1991, 145(2):279-297.

[11] M. Wiercigroch, V. Sin, Experimental study of a symmetrical piecewise base-excited oscillator, J Appl Mech-T ASME, 1998, 65(3):657-663.

[12] V. Sin, M. Wiercigroch, A symmetrically piecewise linear oscillator: design and measurement, P I Mech Eng C-J Mec, 1999, 213(3):241-249.

[13] J. Ing, E. Pavlovskaia, M. Wiercigroch, S. Banerjee, Experimental study of impact oscillator with one-sided elastic constraint, Philos T Roy Soc A, 2008, 366(1866):679705.

[14] J. Ing, E. Pavlovskaia, M. Wiercigroch, S. Banerjee, Bifurcation analysis of an impact oscillator with a one-sided elastic constraint near grazing, Physica D, 2010 3/15, 239(6):312-321.

[15] E. Pavlovskaia, J. Ing, M. Wiercigroch, S. Banerjee, Complex dynamics of bilinear oscillator close to grazing, Int J Bifurcat Chaos, 2010, 20(11):3801-3817.

[16] C. Craig, R. Neilson, J. Penman, The use of correlation dimension in condition monitoring of systems with clearance, J Sound Vib, 2000, 231(1):1-17. 
[17] RLd. Silva, RM. Rubinger, AGd. Oliveira, GM. Ribeiro, Nonlinear dynamics time series analysis of chaotic current oscillations in a semi-insulating GaAs sample, Braz J Phys, 2002, 32(2a):412-414.

[18] R. Hegger, H. Kantz, T. Schreiber, Practical implementation of nonlinear time series methods: The TISEAN package, Chaos, 1999, 9(2):413-435.

[19] JD. Reiss, MB. Sandler, Nonlinear time series analysis of musical signals, In: Proceedings of the international conference on digital audio effects (DAFx-03), London, United Kingdom, 2003, 1-5.

[20] P. Piiroinen, L. Virgin, A. Champneys, Chaos and period-adding, experimental and numerical verification of the grazing bifurcation, J Nonlinear Sci, 2004, 14(4):383-404.

[21] S. Kodba, M. Perc, M. Marhl, Detecting chaos from a time series, Eur J Phys, 2004, 26(1):205.

[22] M. Perc, Nonlinear time series analysis of the human electrocardiogram, Eur J Phys, 2005, 26(5):757.

[23] M. Perc, The dynamics of human gait, Eur J Phys, 2005, 26(3):525.

[24] J. Ing, E. Pavlovskaia, M. Wiercigroch, Dynamics of a nearly symmetrical piecewise linear oscillator close to grazing incidence: Modelling and experimental verification, Nonlinear Dynam, 2006, 46(3):225-238.

[25] J. Ing, Near Grazing Dynamics of Piecewise Linear Oscillators, PhD thesis, University of Aberdeen, 2008.

[26] HD. Abarbanel, R. Brown, J. Sidorowich, L. Tsimring, The analysis of observed chaotic data in physical systems, Rev Mod Phys, 1993, 65:1331-1392.

[27] F. Takens, "Detecting strange attractors in turbulence" in Lecture Notes in Mathematics, DA. Rand, LS. Young, Dynamical Systems and Turbulence, Springer, Warwick, 1981,898:366-381. 
[28] AM. Fraser, HL. Swinney, Independent coordinates for strange attractors from mutual information, Phys Rev A, 1986, 33(2):1134-1140.

[29] MB. Kennel, R. Brown, HD. Abarbanel, Determining embedding dimension for phase-space reconstruction using a geometrical construction, Phys Rev A, 1992, 45(6):3403-3411.

[30] L. Cao, Practical method for determining the minimum embedding dimension of a scalar time series, Physica D, 1997, 110(1):43-50.

[31] T. Schreiber, Detecting and analyzing nonstationarity in a time series using nonlinear cross predictions, Phys Rev Lett, 1997, 78(5):843-846.

[32] DT. Kaplan, L. Glass, Direct test for determinism in a time series, Phys Rev Lett, 1992, 68(4):427-430. 